\title{
USGS St. Petersburg Coastal and Marine Science Center- Research Activities in the U.S. Virgin Islands
}

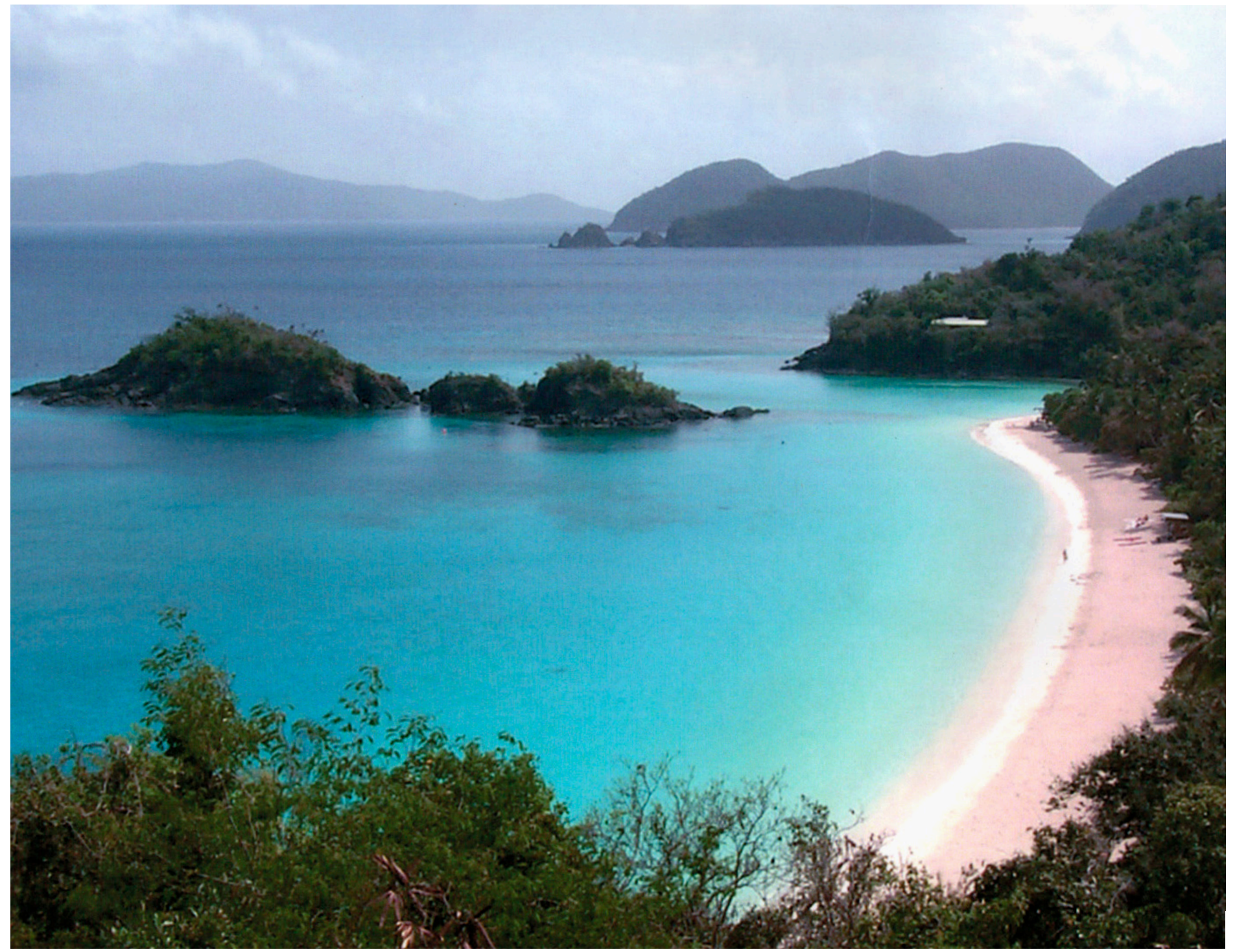

Trunk Bay in the U.S. Virgin Islands.

\section{Current Research Activities}

The U.S. Geological Survey (USGS) St. Petersburg Coastal and Marine Science Center in Florida investigates earth-science processes related to coastal and marine environments as well as to societal implications of natural hazards, resource sustainability, and environmental change. The Center is conducting ongoing research in and around the U.S. Virgin Islands that is providing baseline information for resource management and for assessing the health of and environmental changes to vital ecosystems such as coral reefs. In particular, projects are improving the understanding of coral health, advancing the ability to forecast future changes in coral reef ecosystems, and acquiring topographic data for use in inventorying, monitoring, and conserving coastal and marine environments. 


\section{Assessing Effects of Ocean Acidification and Sea-Level Rise on Coral Reefs}

Coral reefs are vital to the longterm viability of coastal societies, providing economic, recreational, and aesthetic value from which coastal communities thrive. They also provide structural protection to coastlines from high-energy sea states. Coral reefs develop over thousands of years as tropical marine organisms build skeletons of calcium-carbonate $\left(\mathrm{CaCO}_{3}\right)$ to form a three-dimensional structure. This process of calcification can be impacted as ocean chemistry changes. With ever-increasing amounts of carbon dioxide $\left(\mathrm{CO}_{2}\right)$ entering the atmosphere, oceans are absorbing more and more $\mathrm{CO}_{2}$. Part of this $\mathrm{CO}_{2}$ becomes carbonic acid when it dissolves in seawater, lowering $\mathrm{pH}$ levels and increasing seawater acidity. Coupled with greater acidity is a rising sea level also due to increased $\mathrm{CO}_{2}$ in the atmosphere. The rate of sea-level rise is expected to accelerate in the coming century.

Under these changing conditions, USGS scientists are studying whether coral reefs will be able to grow at a rate fast enough to keep up with rising sea level and to sustain reef structure. They are also looking into whether the existing structure will begin to erode away as seawater acidity increases. Scientists are developing comprehensive records of historical and modern reef growth along with calcification rates relative to changing seawater chemistry in order to provide the scientific foundation for predicting future impacts of ocean acidification and sea-level rise on coral reef growth.

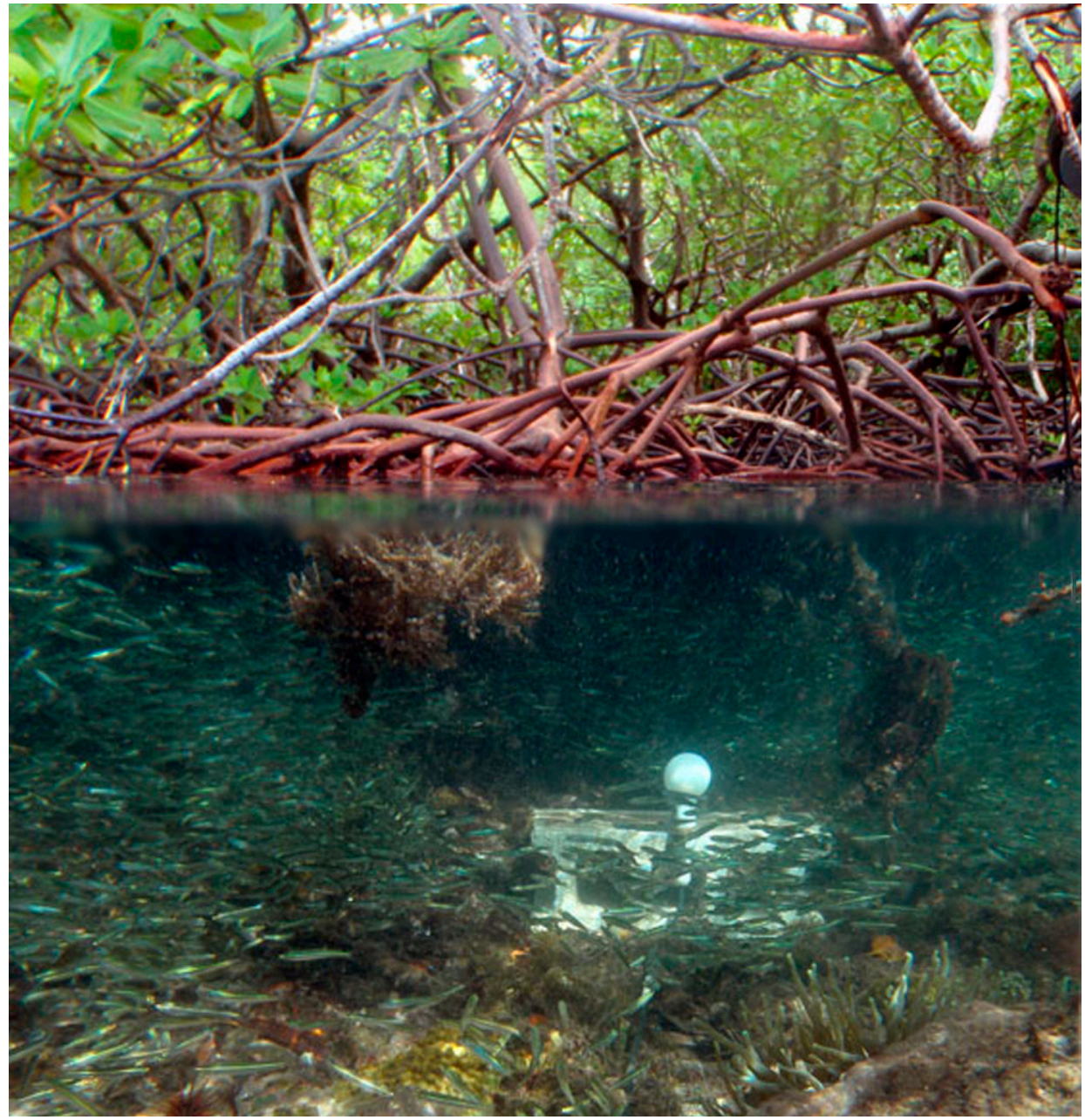

The U.S. Virgin Islands present many unique habitats including complex coral-mangrove environments. Characterization of the coral species, the water chemistry, and the water circulation in this habitat may help in identifying areas resilient to climate change.

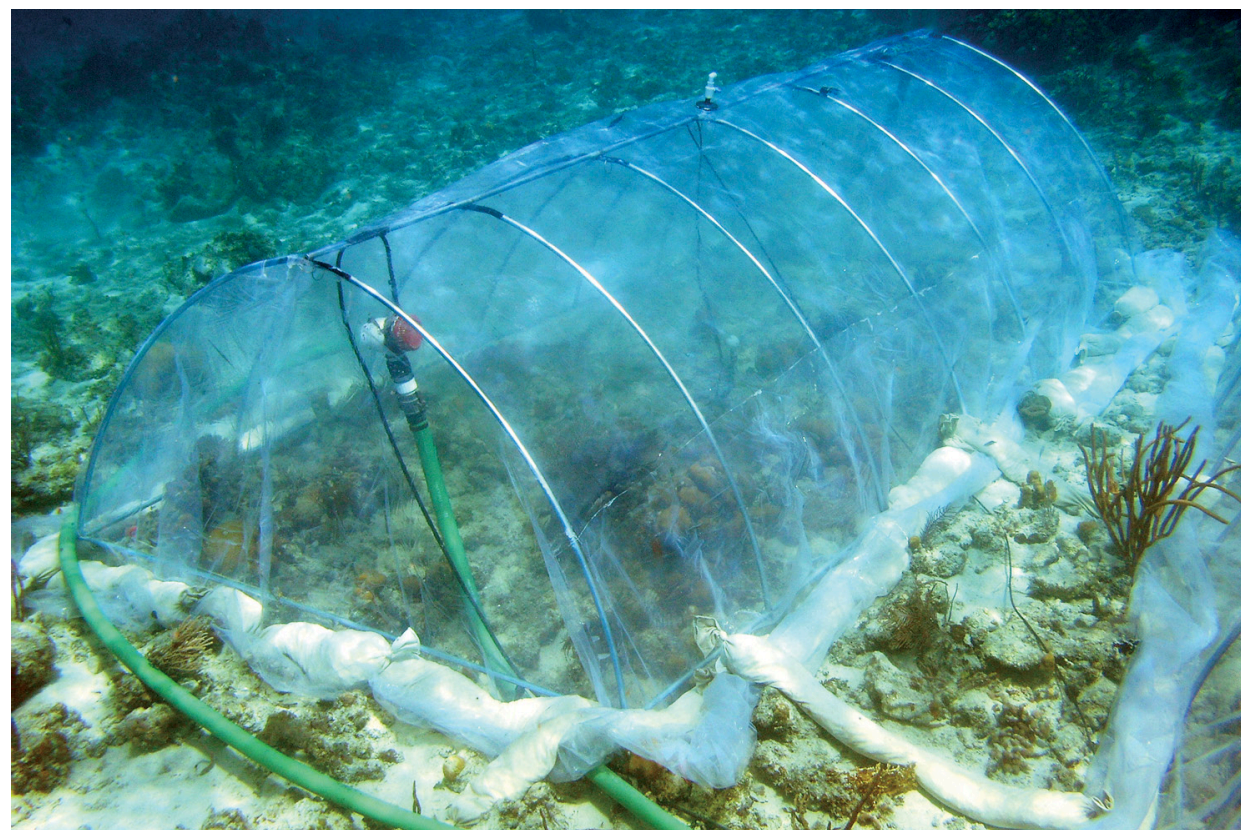

Modern seawater chemistry and growth rates of coral reef habitats are measured using a large (approximately $2.5 \times 5 \times 1.5$ meters) seafloor incubation chamber, the Submersible Habitat for Analyzing Reef Quality (SHARO). The SHARO is also used for in situ experiments that measure growth rates of coral reef habitats subjected to levels of $\mathrm{CO}_{2}$ predicted for the future. 


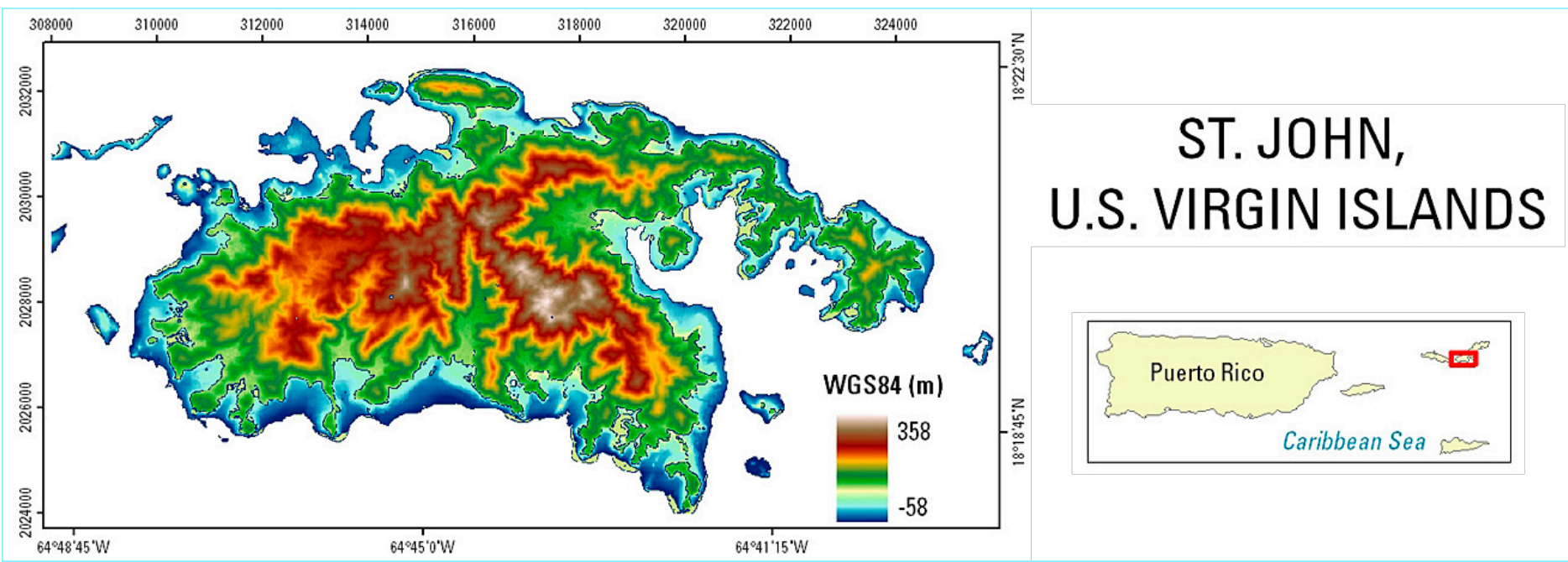

This mosaic image was created from lidar data acquired by the EAARL system during an overflight of St. John, U.S. Virgin Islands, in 2003. The system first acquired surface topography and then submerged nearshore topography to create the high-resolution map.

\section{Acquiring High-Resolution Topographic Data for Resource Management}

High-resolution maps of topography, bathymetry, and shallow seafloor habitat show important features affected by coastal-management decisions.
The mapped information serves as a baseline for evaluating resources and tracking the effectiveness of resource- and conservationmanagement decisions. Scientists at the St. Petersburg Coastal and Marine Science Center also use lidar (light detection and ranging) technology to show the topographic complexity of a barrier island or to monitor changes caused by extreme storms. One technology in particular, the Experimental Advanced Airborne Research Lidar (EAARL) system, is used to capture sub-aerial and submerged topography in the same overflight. Lidar data acquired in
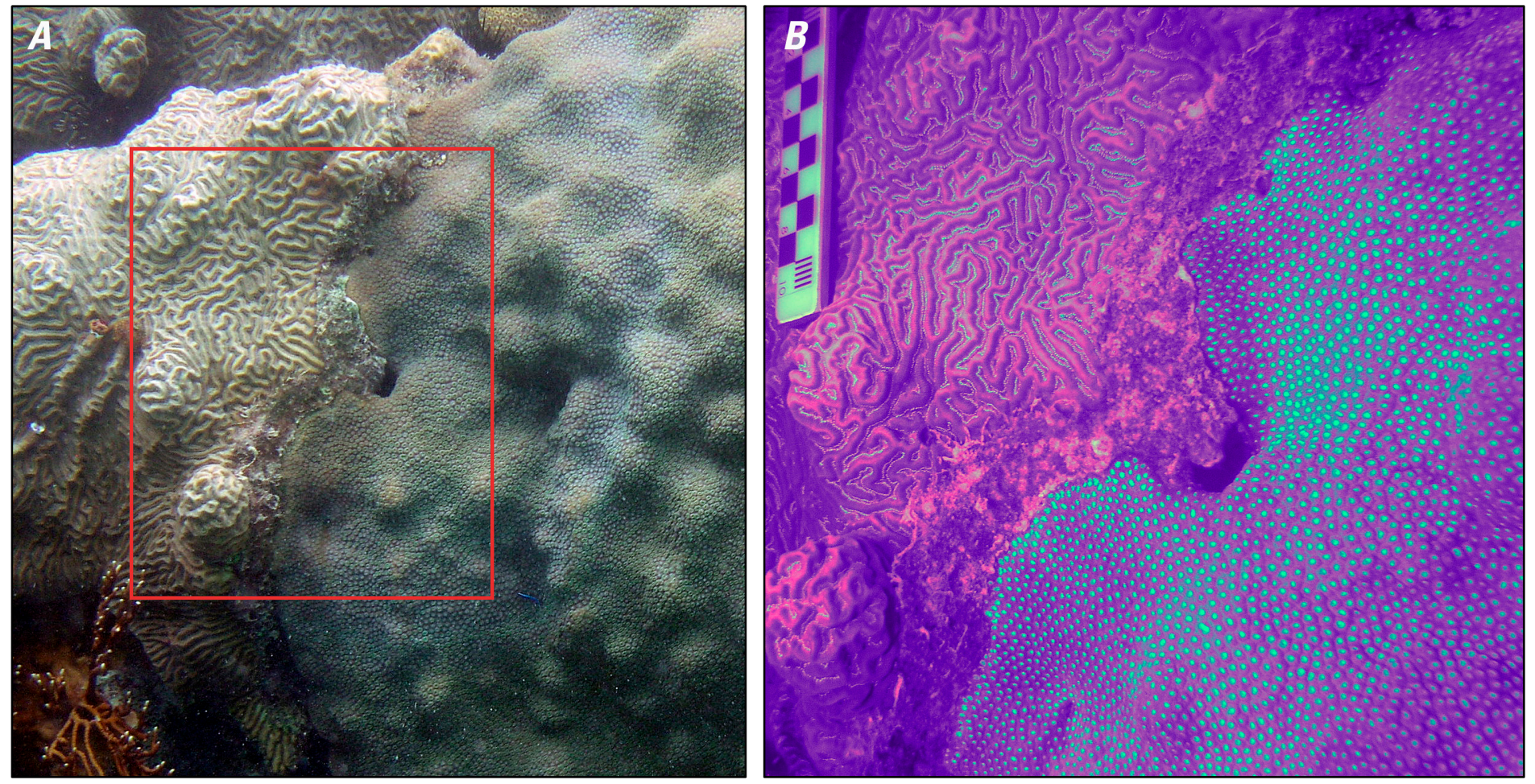

A Under natural lighting, both the knobby brain coral (Diploria clivosa) and the star coral (Montastraea faveolata) are tan or brown. When stimulated to fluoresce with blue light $B$, ridges of the brain coral appear red and its valleys are green. The polyps of the star coral fluoresce green, punctuating a background of red. In both cases, chlorophyll and a green fluorescent protein are responsible for the red and green fluorescent emissions, respectively. Note the border zone between the two corals is dominated by a filamentous alga that fluoresces red due to its chlorophyll content. Ruler squares are 1 centimeter. The red box in $A$ indicates the area shown in $B$. 
collaboration with the National Park Service are being used to inventory and monitor marine benthic communities, barrier-island geomorphology, and vegetated habitats along coastlines. Scientists from the Center are scheduled to return to the U.S. Virgin Islands in 2012 to survey subaerial and submerged topography in collaboration with the National Oceanic and Atmospheric Administration's National Ocean Service.

\section{Using Fluorescence to Diagnose Coral Diseases}

Coral disease, one of the major causes of reef degradation and coral death, has been increasing worldwide since the 1970s, particularly in the Caribbean. Despite increased scientific study, simple questions about the extent of disease outbreaks and the causative agents remain unanswered. USGS scientists are working on better diagnostic techniques to detect onset of disease by using the fluorescent properties within corals. Chlorophyll, found in symbiotic algae called zooxanthellae, which are present in some coral species, has fluorescent properties that can be captured in three colors with a multispectral camera: green, orange, and

\section{Contact Information: \\ Jack Kindinger \\ Director \\ St. Petersburg Coastal and Marine Science Center \\ U.S. Geological Survey St. Petersburg Science Center 600 4th Street South St. Petersburg, FL 33701 \\ PH: (727) 803-8747 x3002 FAX: (727) 803-2031 Email: jkindinger@usgs.gov}

\section{http://coastal.er.usgs.gov/}

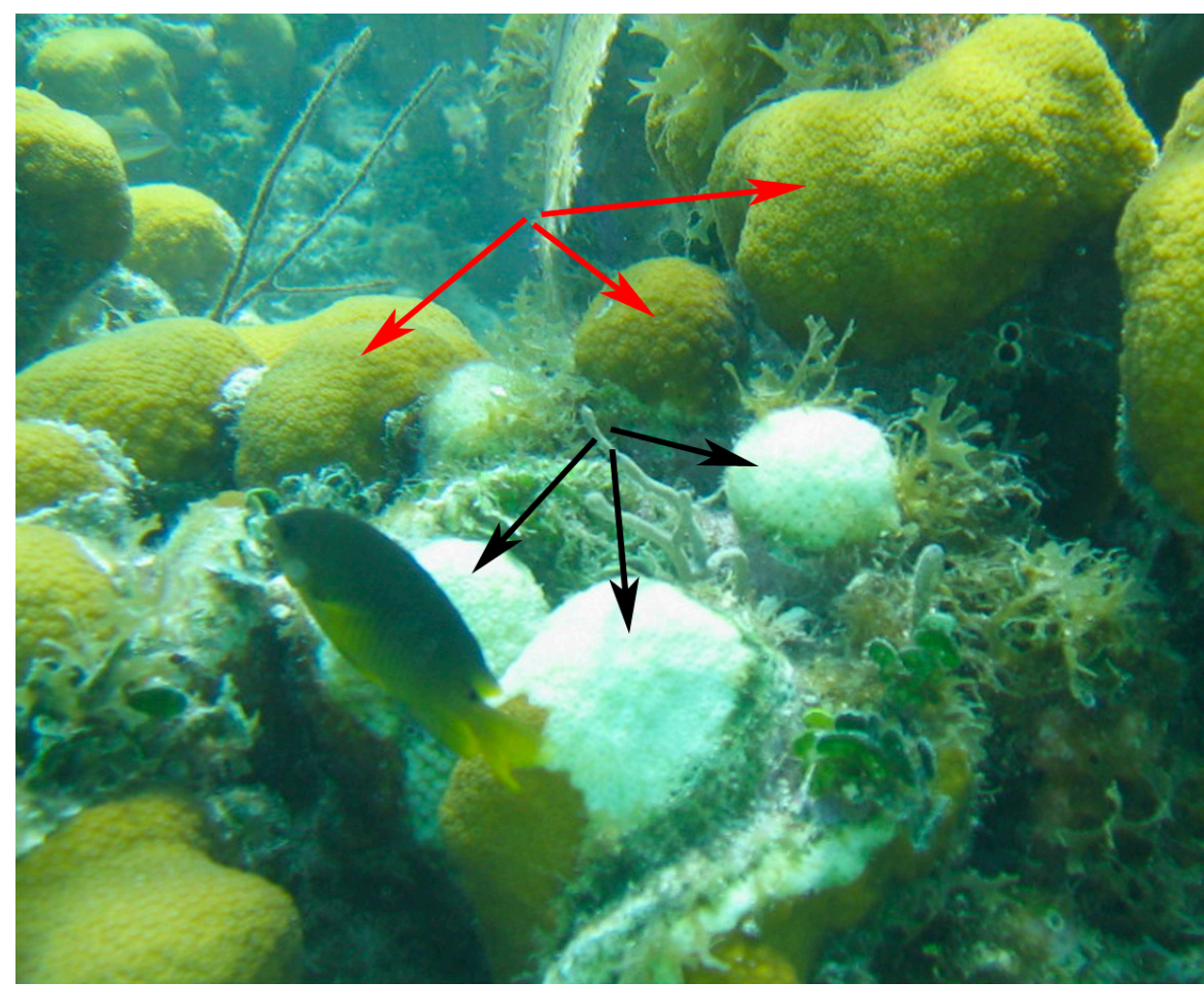

This boulder star coral (Montastraea annularis) is affected by white plague. The white parts of the colony (black arrows) are dead. The disease has rapidly killed the coral, leaving bare white skeleton behind. The apparently healthy parts of the colony are indicated by red arrows.

red. Analyzing this natural variability in fluorescence intensity for a given coral species, and the differences between diseased and healthy specimens, will enable the development of an index relating fluorescence to disease. The use of this diagnostic tool will provide researchers and resource managers with a rapid, non-invasive means to assess coral health.

\section{Characterizing Coral Disease Dynamics}

Although many coral diseases or syndromes are currently being monitored, few causative agents have been identified. Furthermore, such pathogens have not been consistently observed in all corals exhibiting signs of disease. Research has indicated that coral diseases may be secondary opportunistic infections, rather than the result of primary pathogens, making it important to understand the microbial shifts that occur from healthy to diseased corals. This project employed custom-designed microarrays to characterize two coral species at the microbial level. Disease mechanisms are being studied by comparing the microbial profiles of healthy and diseased corals at two sites: Dry Tortugas National Park in the Gulf of Mexico and U.S. Virgin Islands National Park in the Caribbean. This will allow an unprecedented comparison of microbial communities between healthy and diseased corals, between species of coral, and between geographic locations. The microarray data will provide a new baseline of information and may reveal previously unknown patterns underlying the coral diseased state. 